\title{
A Path Following Algorithm for Graph Matching
}

\author{
Mikhail Zaslavskiy ${ }^{1,3,4}$, Francis Bach $^{2}$, and Jean-Philippe Vert ${ }^{1,4}$
}

${ }^{1}$ The Center for Computational Biology, École des Mines de Paris, ParisTech, 35 rue Saint-Honoré, Fontainebleau, France

${ }^{2}$ INRIA-Willow Project, École Normale Supérieure, 45 rue d'Ulm, Paris, France

3 The Center for Mathematical Morphology, École des Mines de Paris, ParisTech, 35 rue Saint-Honoré, Fontainebleau, France

${ }^{4}$ Institut Curie, Section Recherche, INSERM U900, Paris, France

\begin{abstract}
We propose a convex-concave programming approach for the labelled weighted graph matching problem. The convex-concave programming formulation is obtained by rewriting the graph matching problem as a least-square problem on the set of permutation matrices and relaxing it to two different optimization problems: a quadratic convex and a quadratic concave optimization problem on the set of doubly stochastic matrices. The concave relaxation has the same global minimum as the initial graph matching problem, but the search for its global minimum is aslo a complex combinatorial problem. We therefore construct an approximation of the concave problem solution by following a solution path of the convex-concave problem obtained by linear interpolation of the convex and concave formulations, starting from the convex relaxation. The algorithm is compared with some of the best performing graph matching methods on three datasets: simulated graphs, QAPLib and handwritten chinese characters.
\end{abstract}

\section{Introduction}

The graph matching problem consists in finding a correspondence between vertices of two given graphs which is optimal in a certain sense 1 . This problem plays a very important role in various fields of pattern recognition like optical character recognition [1, image analysis (2D and 3D) [2], or bioinformatics [4.

Because of the combinatorial nature of the problem, it is very hard to solve it exactly for large graphs; however some methods based on incomplete enumeration may be applied to search for an exact optimal solution in the case of small or sparse graphs. An alternative is to use approximate algorithms which are supposed to be much more scalable, but only find an approximate solution. This group is represented by two classes of methods: spectral methods [5, 6, 2, 7 , and methods which work directly with graph adjacency matrices and typically involve a relaxation of the discrete optimization problem [8, 9, 10,3.

An interesting instance of the graph matching problem is the matching of labeled graphs. If there is also a similarity measure between labels, then we

\footnotetext{
${ }^{1}$ Usually, a correspondence is optimal if it aligns graph structures in the best way, but other criteria are possible as well.
} 
search a correspondence which matches not only the structures of the graphs but also vertices with similar labels. Some widely used approaches only use the information about similarities between graph labels 11, some try to combine information on graph structures and vertex similarities [10,4. We do not discuss here the various graph matching algorithms, a good review of graph matching algorithms may be found in [12].

In this article, we propose an approximate methods for labeled weighted graph matching. This method is based on a formulation of the labeled graph matching problem as a quadratic assignment problem (QAP) over the set of permutation matrices, where the quadratic term encodes the structural compatibility and an additional linear term encodes vertex compatibilities. We propose two relaxations of this problem, resulting in one quadratic convex and one quadratic concave optimization problem on the set of doubly stochastic matrices. While the concave relaxation has the same global minimum as the initial QAP, it also does not have an efficient optimization algorithm. We find a local minimum of this problem by following a solution path of a family of convex-concave optimization problems, obtained by linearly interpolating the convex and concave relaxations. Starting from the convex formulation with a unique local (and global) minimum, the solution path leads to a local optimum of the concave relaxation. We perform an extensive comparison of this PATH algorithm with several state-of-the-art matching methods on small simulated graphs and various QAP benchmarks, and show that it consistently provides state-of-the-art performances while scaling to graphs of up to a few thousands vertices for a modern computer. We further illustrate the use of the algorithm on the matching of handwritten chinese characters. Additional simulations may be found in 13 .

\section{Problem Description}

A graph $G=(V, E)$ of size $N$ is defined by a finite set of vertices $V=\{1, \ldots, N\}$ and a set of edges $E \subset V \times V$. We consider weighted undirected graphs with no self-loop, i.e., all edges $(i, j)$ have an associated positive real value $w(i, j)=$ $w(j, i)$ and $w(i, i)=0 \forall i, j \in V$. Each such graph can be equivalently represented by a symmetric adjacency matrix $A$ where $A_{i j}=w(i, j)$.

Given two graphs $G$ and $H$ with the same number of vertices $N 2$, the problem of matching $G$ and $H$ consists in finding a correspondence between vertices of $G$ and vertices of $H$ which aligns $G$ and $H$ in some optimal way. The correspondence between vertices may be defined by a permutation matrix $P, P_{i j}$ is equal to 1 if the $i$-th vertex of $G$ is matched to the $j$-th vertex of $H$, and 0 otherwise. After applying the permutation defined by $P$ to the vertices of $H$ we obtain a new graph isomorphic to $H$ which we denote by $P(H)$. The adjacency matrix of the permuted graph, $A_{P(H)}$, is simply obtained from $A_{H}$ by the equality $A_{P(H)}=P A_{H} P^{T}$.

In order to assess whether a permutation $P$ defines a good matching between the vertices of $G$ and those of $H$, a quality criterion must be defined. We focus

${ }^{2}$ Otherwise the smallest may be completed with dummy nodes. 
in this paper on measuring the discrepancy between the graphs after matching of edges which are present in one graph and not in the other one:

$$
F(P)=\left\|A_{G}-A_{P(H)}\right\|_{F}^{2}=\left\|A_{G}-P A_{H} P^{T}\right\|_{F}^{2},
$$

where \|\|$_{F}$ is the Frobenius matrix norm. Therefore, the problem of graph matching can be reformulated as the problem of minimization of $F(P)$ over the set of permutation matrices.

An interesting generalization of the graph matching problem is the problem of labeled graph matching. Here each graph has associated labels to all its vertices and the objective is to find an alignment that fits well graph labels and graph structures at the same time. If we let $C_{i j}$ denote the cost of fitness between $i$-th vertex of $G$ and $j$-th vertex of $H$ then the matching problem based only on label comparison can be formulated as follows

$$
\min _{P} \operatorname{tr}\left(C^{T} P\right)=\sum_{i=1}^{N} \sum_{j=1}^{N} C_{i j} P_{i j}=\sum_{i=1}^{N} C_{i, P(i)} .
$$

A natural way of unifying of (2) and (11) is a linear combination

$$
\min _{P}\left\{(1-\alpha) F(P)+\alpha \operatorname{tr}\left(C^{T} P\right)\right\} .
$$

In the next section we describe our new algorithm which is based on the technique of convex-concave relaxation of the initial problem (11).

\section{Convex-Concave Relaxation}

The criterion of graph matching problem we consider is (10). Since permutation matrices are also orthogonal matrices, we can rewrite $F(P)$ on $\mathcal{P}$ as follows:

$$
F_{0}(P)=\left\|A_{G}-P A_{H} P^{T}\right\|_{F}^{2}=\left\|\left(A_{G}-P A_{H} P^{T}\right) P\right\|_{F}^{2}=\left\|A_{G} P-P A_{H}\right\|_{F}^{2} .
$$

The graph matching problem is then the problem of minimizing $F_{0}(P)$ over $\mathcal{P}$, which we call GM:

$$
\text { GM: } \min _{P \in \mathcal{P}} F_{0}(P) \text {. }
$$

A first relaxation of $\mathbf{G M}$ is obtained by expanding the convex quadratic function $F_{0}(P)$ on the set of doubly stochastic matrices $\mathcal{D}$ :

$$
\text { QCV: } \min _{P \in \mathcal{D}} F_{0}(P) \text {. }
$$

The QCV problem may be solved in polynomial time (this is a convex quadratic minimization problem) by the Frank-Wolfe algorithm [14, but the optimal value usually does not belong to the set of the extreme points of $\mathcal{D}$ so we have to use the approximation: $\arg \min _{\mathcal{P}} F(P) \approx \Pi_{\mathcal{P}} \arg \min _{\mathcal{D}} F(P)$. The projection $\Pi_{\mathcal{P}}$ may be made by the Hungarian algorithm in $O\left(N^{3}\right)$, but the problem is that if $\arg \min _{\mathcal{D}} F(P)$ is far from $\mathcal{P}$ then the approximation quality may be poor. 
We now present a second relaxation of GM, resulting in a concave minimization problem. For that purpose, let us introduce the diagonal degree matrix $D$ of an adjacency matrix $A$, which is the diagonal matrix with entries $D_{i i}=d(i)=\sum_{i=1}^{N} A_{i j}$ for $i=1, \ldots, N$, as well as the Laplacian matrix $L=D-A$. A having only nonnegative entries, it is well-known that the Laplacian matrix is positive semidefinite [15]. We can now rewrite $F(P)$ as follows:

$$
\begin{aligned}
& F_{0}(P)=\left\|A_{G} P-P A_{H}\right\|_{F}^{2}=\left\|\left(D_{G} P-P D_{H}\right)-\left(L_{G} P-P L_{H}\right)\right\|_{F}^{2} \\
& =\left\|D_{G} P-P D_{H}\right\|_{F}^{2}-2 \operatorname{tr}\left[\left(\mathrm{D}_{\mathrm{G}} \mathrm{P}-\mathrm{PD}_{\mathrm{H}}\right)^{\mathrm{T}}\left(\mathrm{L}_{\mathrm{G}} \mathrm{P}-\mathrm{PL}_{\mathrm{H}}\right)\right]+\left\|\mathrm{L}_{\mathrm{G}} \mathrm{P}-\mathrm{PL}\right\|_{\mathrm{F}}^{2} .
\end{aligned}
$$

After some transformations $F_{0}(P)$ may be rewritten as

$$
F_{0}(P)=-\operatorname{tr}(\Delta P)+\operatorname{tr}\left(L_{G}^{2}\right)+\operatorname{tr}\left(L_{H}^{2}\right)-2 \operatorname{vec}(P)^{T}\left(L_{H} \otimes L_{G}\right) \operatorname{vec}(P),
$$

where we introduced the matrix $\Delta_{i, j}=\left(D_{H}(j, j)-D_{G}(i, i)\right)^{2}$ and we used $\otimes$ to denote the Kronecker product of two matrices. Let denote $F_{1}(P)$ the function defined in (7) expanded on $\mathcal{D}$. Since graph Laplacian matrices are positive semidefinite, the matrix $L_{H} \otimes L_{G}$ is also positive semidefinite as a Kronecker product of two symmetric positive semi-definite matrices [16. Therefore the function $F_{1}(P)$ is concave on $\mathcal{D}$, and we obtain a concave relaxation of the graph matching problem:

$$
\text { QCC: } \min _{P \in \mathcal{D}} F_{1}(P) \text {. }
$$

Interestingly, the global minimum of a concave function is necessarily at one of the extreme points of the convex set where it is minimized, so the minimum of $F_{1}(P)$ on $\mathcal{D}$ is in fact in $\mathcal{P}$. At this point, we have obtained two relaxations of GM as optimization problems on $\mathcal{D}$ : the first one is the convex minimization problem QCV (6), which can be solved efficiently but leads to a solution in $\mathcal{D}$ that must then be projected onto $\mathcal{P}$, and the other is the concave minimization problem QCC (8) which can not be solved efficiently but has the same solution as the initial combinatorial problem GM.

We propose to approximately solve QCC by tracking a path of local minima over $\mathcal{D}$ of a sequence of functions that linearly interpolate between $F_{0}(P):=$ $F(P)$ and $F_{1}(P)$, namely:

$$
F_{\lambda}(P)=(1-\lambda) F_{0}(P)+\lambda F_{1}(P),
$$

for $0 \leq \lambda \leq 1$. For all $\lambda \in[0,1], F_{\lambda}$ is a quadratic function. We recover the convex function $F_{0}$ for $\lambda=0$, and the concave function $F_{1}$ for $\lambda=1$. Our method searches sequentially local minima of $F_{\lambda}$, where $\lambda$ moves from 0 to 1 . More precisely, we start at $\lambda=0$, and find the unique local minimum of $F_{0}$ (which is in this case its unique global minimum) by any classical QP solver. Then, iteratively, we find a local minimum of $F_{\lambda+d \lambda}$ given a local minimum of $F_{\lambda}$ by performing a local optimization of $F_{\lambda+d \lambda}$ starting from the local minimum of $F_{\lambda}$, using for example the Frank-Wolfe algorithm. Repeating this iterative process for $d \lambda$ small enough we obtain a path of solutions $P^{*}(\lambda)$, where $P^{*}(0)=$ $\arg \min _{P \in \mathcal{D}} F_{0}(P)$ and $P^{*}(\lambda)$ is a local minimum of $F_{\lambda}$ for all $\lambda \in[0,1]$. Noting 
that any local minimum of the convex function $F_{1}$ on $\mathcal{D}$ is in $\mathcal{P}$, we finally output $P^{*}(1) \in \mathcal{P}$ as an approximate solution of $\mathbf{G M}$.

The pseudo-code for this PATH algorithm is presented below.

\section{Initialization:}
(a) $\lambda:=0$
(b) $P^{*}(0)=\arg \min F_{0}$ - standard QP solver.

2. Cycle over $\lambda$ :

do
(a) $\lambda_{\text {new }}:=\lambda+d \lambda$
(b) if $\left|F_{\lambda_{\text {new }}}\left(P^{*}(\lambda)\right)-F_{\lambda_{\text {new }}}\left(P^{*}(\lambda)\right)\right|<\epsilon$ then $\quad \lambda=\lambda_{\text {new }}$
else
$P^{*}\left(\lambda_{\text {new }}\right)=\arg \min F_{\lambda_{\text {new }}}$ is found by Frank-Wolfe starting from $P^{*}(\lambda)$ $\lambda=\lambda_{\text {new }}$
endif
while $\lambda<1$
3. Output: $P^{\text {out }}:=P^{*}(1)$

Of course, in spite of these justifications the PATH algorithm only gives an approximation of the global minimum in the general case. For a more detailed analysis, see 13 .

If we match two labeled graphs, then we may increase the performance of our method by using information on pairwise similarities between their nodes:

$$
\min _{P} F_{\lambda}^{\alpha}(P)=\min _{P}(1-\alpha) F_{\lambda}(P)+\alpha \operatorname{tr}\left(C^{T} P\right) .
$$

The advantage of the last formulation is that $F_{\lambda}^{\alpha}(P)$ is just $F_{\lambda}(P)$ with an additional linear term. Therefore we can use the same algorithm for $F_{\lambda}^{\alpha}(P)$ optimization that we have used before for $F_{\lambda}(P)$.

\section{Results}

Synthetic examples. In this section we compare the proposed algorithm with some classical methods on the example of artificially generated graphs. Our choice of random graph types is based on [17. Each type of random graphs is defined by the distribution function of node degree, we consider three distributions: binomial (bin), exponential (exp) and power law (pow). If we are interested in isomorphic graph matching then we compare just the initial graph and its randomly permuted copy. To test the matching of non-isomorphic graphs, we add randomly $\sigma_{n} N_{E}$ edges to the initial graph and to its permitted copy, where $N_{E}$ is the number of edges in the original graph, and $\sigma_{n}$ is the noise level.

The first series of experiments are experiments on small size graphs $(\mathrm{N}=8)$, here we are interested in comparison of PATH algorithm, QCV approach (6), Umeyama spectral algorithm "U" [5], a linear programming approach LP [8] and exhaustive search OPT which is feasible for the small size graphs. The results are presented on the top line in Figure 1. The second row of Figure 1 presents 

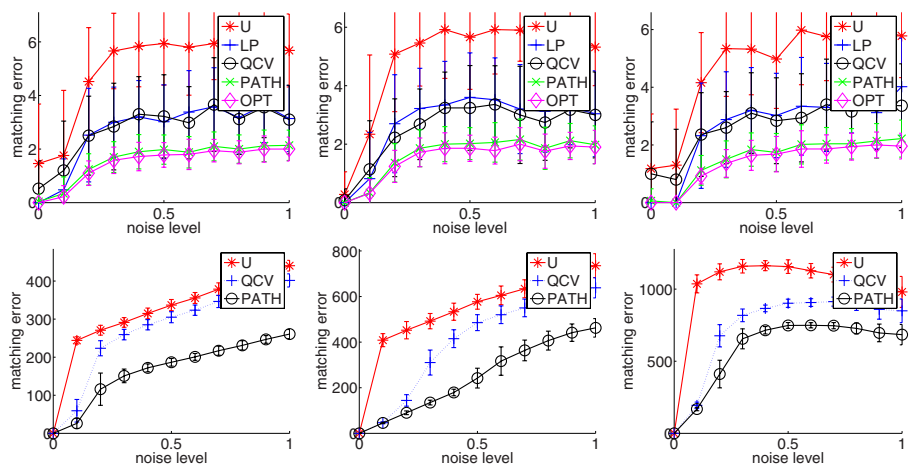

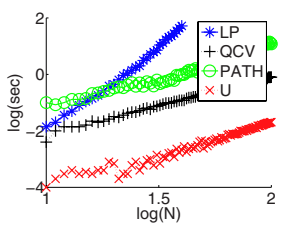

(a) bin

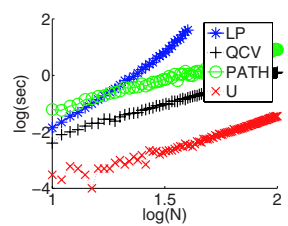

(b) $\exp$

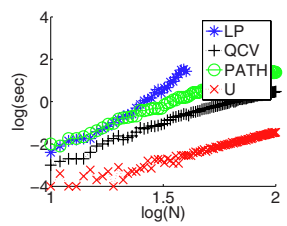

(c) pow

Fig. 1. Precision and timing of graph matching algorithms

results of experiments on large graphs $(\mathrm{N}=100)$. In all cases PATH algorithm works much better than all other algorithms. Note, that the application of solution path is useful (PATH algorithms works much better than QCV). A very remarkable thing is that PATH solution is very close to the global minimum. The third row presents the algorithm complexity (i.e the time of computing time as a function of graph size), $\mathbf{U}$ algorithm is known to be one of the fastest graph matching algorithm, PATH and QCV show the same order of complexity (slope of the corresponding curves $\approx 3$ ), complexity of $\mathbf{L P}$ is $O\left(N^{7}\right)$. All results are coherent with theoretical values of algorithm complexities.

QAP benchmark library. The problem of graph matching may be considered as a particular case of the quadratic assignment problem (QAP). 10] proposed the QPB graph matching algorithm and tested it on matrices from the QAP benchmark library (see [18), as well as graduated assignment algorithm GRAD 9] and Umeyama. Results of PATH application to the same matrices are presented in the table (1). PATH outperforms QPB, GRAD and U almost in all cases.

Recognition of handwritten chinese characters. Another example that we consider in this paper is the problem of handwritten chinese character recognition from the ETL9B database. We use a score of optimal matching as a similarity measure in KNN for character classification. Here we compare the performance of four methods: linear support vector machine (SVM), SVM with gaussian kernel, KNN based on score of shape context matching and K-nearest neighbor (KNN) based on score of graph matching. As score we use just the value of the objective 
Table 1. QAP benchmark library

\begin{tabular}{|l|l|l|l|l|l|}
\hline QAP & MIN & PATH & QPB & GRAD & U \\
\hline \hline chr12c & 11156 & $\mathbf{1 8 0 4 8}$ & 20306 & 19014 & 40370 \\
\hline chr15a & 9896 & $\mathbf{1 9 0 8 6}$ & 26132 & 30370 & 60986 \\
\hline chr15c & 9504 & $\mathbf{1 6 2 0 6}$ & 29862 & 23686 & 76318 \\
\hline chr20b & 2298 & $\mathbf{5 5 6 0}$ & 6674 & 6290 & 10022 \\
\hline chr22b & 6194 & $\mathbf{8 5 0 0}$ & 9942 & 9658 & 13118 \\
\hline esc16b & 292 & 300 & $\mathbf{2 9 6}$ & 298 & 306 \\
\hline rou12 & 235528 & $\mathbf{2 5 6 3 2 0}$ & 278834 & 273438 & 295752 \\
\hline rou15 & 354210 & 391270 & $\mathbf{3 8 1 0 1 6}$ & 457908 & 480352 \\
\hline rou20 & 725522 & $\mathbf{7 7 8 2 8 4}$ & 804676 & 840120 & 905246 \\
\hline tai10a & 135028 & $\mathbf{1 5 2 5 3 4}$ & 165364 & 168096 & 189852 \\
\hline tai15a & 388214 & $\mathbf{4 1 9 2 2 4}$ & 455778 & 451164 & 483596 \\
\hline tai17a & 491812 & $\mathbf{5 3 0 9 7 8}$ & 550852 & 589814 & 620964 \\
\hline tai20a & 703482 & $\mathbf{7 5 3 7 1 2}$ & 799790 & 871480 & 915144 \\
\hline tai30a & 1818146 & $\mathbf{1 9 0 3 8 7 2}$ & 1996442 & 2077958 & 2213846 \\
\hline tai35a & 2422002 & $\mathbf{2 5 5 5 1 1 0}$ & 2720986 & 2803456 & 2925390 \\
\hline tai40a & 3139370 & $\mathbf{3 2 8 1 8 3 0}$ & 3529402 & 3668044 & 3727478 \\
\hline
\end{tabular}

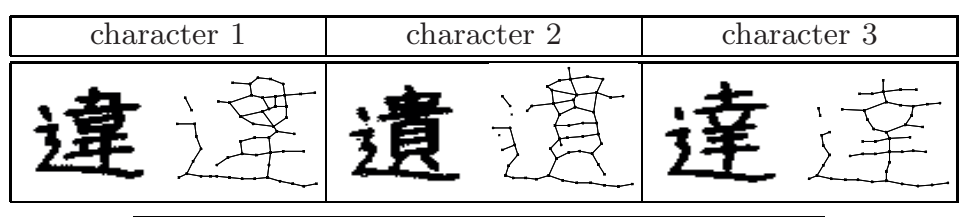

\begin{tabular}{|l|c|c|}
\hline Method & $C V$ & $S T D$ \\
\hline \hline Linear SVM & 0.377 & \pm 0.090 \\
SVM with gaussian kernel & 0.359 & \pm 0.076 \\
KNN $(\lambda=1)$ : shape context & 0.399 & \pm 0.081 \\
KNN $(\lambda=0.4)$ & 0.248 & \pm 0.075 \\
KNN $(\lambda=0)$ : pure graph matching & 0.607 & \pm 0.072 \\
\hline
\end{tabular}

Fig. 2. Chinese characters. $(C V, S T D)$ - mean and standard deviation of test error over cross-validation runs (five folds, 50 repetition)

function (9) in the optimal point. We have selected three chinese characters which are the most difficult to distinguish. Examples of these characters as well as examples of extracted graphs are presented in Figure2. In SVM based algorithms we use directly the values of image pixels (so each image is represented by a binary vector), in graph matching algorithm we use binary adjacency matrices of extracted graphs and shape context matrices (see [11]). Our data set consist of 50 exemplars (images) of each class. To compare different methods we use the cross validation error (five folds). Complete results may be found in Figure 2 . 


\section{Conclusion}

We have presented the PATH algorithm, a new method for graph matching based on convex-concave relaxations of the initial integer programming problem. It obtained very promising results in all experiments against state-of-the-art methods, and has the same empirical complexity as the fastest graph matching algorithms. It furthermore allows to easily integrate the information on label similarities. In future works it will be interesting to investigate other choices of convex and concave relaxations, and to generalize the approach to directed grephs, i.e., asymmetric adjacency matrices, which could provide new methods for the general QAP.

\section{References}

1. Filatov, A., Gitis, A., Kil, I.: Graph-based handwritten digit string recognition. In: Third International Conference on Document Analysis and Recognition (ICDAR 1995), pp. 845-848 (1995)

2. Carcassoni, M., Hancock, R.E.: Spectral correspondance for point pattern matching. Pattern Recognition 36, 193-204 (2002)

3. Schellewald, C., Schnor, C.: Probabilistic subgraph matching based on convex relaxation. Lecture notes in computer science, pp. 171-186. Springer, Heidelberg (2005)

4. Singh, R., Xu, J., Berger, B.: Pairwise global alignment of protein interaction networks by matching neighborhood topology. Research in Computantional Molecular Biology 4453, 16-31 (2007)

5. Umeyama, S.: An eigendecomposition approach to weighted graph matching problems. Transaction on pattern analysis and machine intelligence 10 (1988)

6. Shapiro, L., Brady, J.: Feature-based correspondance: an eigenvector approach. Image and vision computing 10, 283-288 (1992)

7. Caelli, T., Kosinov, S.: An eigenspace projection clustering method for inexact graph matching. TPAMI 24 (2004)

8. Almohamad, H., Duffuaa, S.O.: A linear programming approach for the weighted graph matching problem. TPAMI 15 (1993)

9. Gold, S., Rangarajan, A.: A graduated assignment algorithm for graph matching. Transaction on pattern analysis and machine intelligence 18 (1996)

10. Cremers, D., Kohlberger, T., Schnor, C.: Evaluation of convex optimization techniques for the graph-matching problem in computer vision. Patter Recogn. 2191 (2001)

11. Belongie, S., Malik, J., Puzicha, J.: Shape matching and object recognition using shape contexts. Transaction on pattern analysis and machine intelligence 24 (2002)

12. Conte, D., Foggia, P., Sansone, C., Vento, M.: Thirty years of graph matching in pattern recognition. International journal of pattern recognition and AI 18, 265298 (2004)

13. Zaslavskiy, M., Bach, F., Vert, J.P.: Path following algorithm for graph matching problem. arXiv:0801.3654v1 (2008)

14. Frank, M., Wolfe, P.: An algorithm for quadratic programming. Naval Research Logistics Quarterly 3, 95-110 (1956) 
15. Chung, F.R.K.: Spectral Graph Theory. Americal Mathematical Society (1997)

16. Golub, G.H., Loan, C.F.V.: Matrix computations, 3rd edn. Johns Hopkins University Press, Baltimore (1996)

17. Newman, M.E.J., Strogatz, S.H., Watts, D.J.: Random graphs with arbitrary degree distributions and their applications. PHYSICAL REVIEW 64 (2001)

18. Cela, E.: Qaudratuc assignment problem library (2007), http://www.opt.math. tu-graz.ac.at/qaplib/ 